\section{SCIENCE CHINA \\ Physics, Mechanics \& Astronomy}

\title{
Beyond the operator algebra-To be nonlocal realism of quantum mechanics
}

\author{
Zhi-Han Zhu* \\ Wang Da-Heng Collaborative Innovation Center for Science of Quantum Manipulation \& Control, Heilongjiang Provincial Key Laboratory of \\ Quantum Manipulation \& Control, Harbin University of Science and Technology, Harbin 150080, China \\ Received January 4, 2018; accepted January 8, 2018; published online January 29, 2018 \\ Citation: Z. H. Zhu, Beyond the operator algebra-To be nonlocal realism of quantum mechanics, Sci. China-Phys. Mech. Astron. 61, 050331 (2018), https:// \\ doi.org/10.1007/s11433-018-9163-8
}

In 1905, Albert Einstein presented his milestone theory of photons in his paper titled "On a Heuristic Viewpoint Concerning the Production and Transformation of Light" based on Max Planck's black-body radiation theory. The groundbreaking theory successfully solved the paradox between Maxwell's wave theory of light and the experimental finding on photoelectric effect, propelling the concept of wave-particle duality and granting Einstein with his solo Nobel Prize in 1921. Later, a group of physicists and mathematicians developed a wavefunction formulation based on operator algebra to describe quantum theory. This powerful mathematical abstraction, the greatest theoretical invention in human history, enables us to calculate and predict physical process in the microscopic world that people cannot intuitively understand, such as atomic energy levels, electron tunneling, and photon trajectories. Through this drastic paradigm shift, using wave theory to describe a particle system, the first quantum revolution was directly produced, laying the foundation of modern science and technology. This shift, however, posed a profound question about abstraction and reality, i.e., how to understand a dynamical process on Hilbert space, especially for cases involving the concept of superposition [1].

Are quantum states real? Today, nearly a century after the birth of quantum mechanics, a second quantum revolution is occurring [2] and the theory is extending into more general

*Corresponding author (email: zhuzhihan@hrbust.edu.cn) regimes [3]; however, this old question persists. Bohr and Schrödinger, representatives of the Copenhagen interpretation, first may have thought a quantum particle really existed in the form of its wavefunction. However, when Einstein, Podolsky, and Rosen [4] showed that the cost of reality requires a superluminal connection between two real systems, Bohr and Schrödinger had to abandon that idea and concluded that no elementary phenomenon is a phenomenon until it is a registered phenomenon. Despite this, Einstein insisted that physics should look for "really existing objects" or to be local realism theory.

Over the next few decades, the nonlocal property of the quantum world was repeatedly verified via experiments, while the debate on reality always was a controversial topic. In various delayed-choice experiments dedicated to this topic [5], Copenhagen interpretation claimed that the photons passing through the double slits have no definite nature until they are measured and that wavefunctions provide only "a catalog of knowledge". In contrast, based on Einstein's famous query, "Do you really think the moon is not there if you are not looking at it?" the determinists argued that the past of photons should be realistic and deterministic prior to the detection. Despite the results obtained 5 years ago in various delayed-choice experiments seem to have rejected the realistic interpretation, the recently observed wave-particle superposition and disconnected propagation history make the long-standing debate more intense [6,7]. Owing to 
the recent advance in science of structured light $[8,9]$, the new finding of the subluminal feature of twisted light enables us to see the real past of photons after being registered; consequently, the nonlocal reality of the quantum world was finally confirmed in 2017 [10,11].

Almost the same time, Long et al. [12] from Tsinghua University reported an encounter-delayed-choice (EDC) experiment based on the idea of realistic interpretation of a wavefunction in quantum mechanics, in which the nonlocality and reality of the wavefunction is verified. More interestingly, in the EDC experiment, by inserting the second beam splitter (BS) of a Mach-Zehnder interferometer when the two sub waves (pulses) converge in the middle, first, a path-superposed photon (wave nature) partially (or weakly) collapses into distinguishable-path bases (particle nature); second, it is measured with an identical-path base (wave nature). That is to say, photons can first behave as a particle and then behave as a wave. This staggering result indicates that the evolution of the whole system is deterministic through the time axis. Specifically, a photon is first converted into a superposition of two sub waves via BS-1; then, the photon weakly couples with the experimental apparatus and the two sub waves together evolve into a collapse-or-not superposition state when the front part of the wave pulse is detected in particle nature; lastly, the collapse-or-not superposition is observed in the wave nature when BS-2 is inserted.
More remarkably, in addition to the nonlocal realism, the deterministic evolution history revealed in this brilliant work deepened our understanding in the complementary concepts of abstraction and reality. The results indicate that the quantum states described by wavefunctions are real and that the abstract-real boundary in the physical world is blurry. Namely, start now, it is more valuable to ask whether we live in Hilbert space rather than to doubt to be or not to be real.

1 C. P. Sun, Physics 46, 481 (2017).

2 J. P. Dowling, and G. J. Milburn, Philos. Trans. R. Soc. A 361, 1655 (2003).

3 C. M. Bender, D. C. Brody, and H. F. Jones, Phys. Rev. Lett. 89, 270401 (2002).

4 A. Einstein, B. Podolsky, and N. Rosen, Phys. Rev. 47, 777 (1935).

5 X. Ma, J. Kofler, and A. Zeilinger, Rev. Mod. Phys. 88, 015005 (2016), arXiv: 1407.2930

6 J. S. Tang, Y. L. Li, X. Y. Xu, G. Y. Xiang, C. F. Li, and G. C. Guo, Nat. Photon 6, 600 (2012).

7 A. Danan, D. Farfurnik, S. Bar-Ad, and L. Vaidman, Phys. Rev. Lett. 111, 240402 (2013).

8 F. Bouchard, J. Harris, H. Mand, R. W. Boyd, and E. Karimi, Optica 3, 351 (2016).

9 D. Giovannini, J. Romero, V. Potoček, G. Ferenczi, F. Speirits, S. M. Barnett, D. Faccio, and M. J. Padgett, Science 347, 857 (2015), arXiv: 1411.3987

10 Z. Y. Zhou, Z. H. Zhu, S. L. Liu, Y. H. Li, S. Shi, D. S. Ding, L. X. Chen, W. Gao, G. C. Guo, and B. S. Shi, Sci. Bull. 62, 1185 (2017).

11 G. L. Long, Sci. Bull. 62, 1355 (2017).

12 G. L. Long, W. Qin, Z. Yang, and J. L. Li, Sci. China-Phys. Mech. Astron. 61, 030311 (2018). 